\title{
A CORRELATION RESULT FOR NONSTATIONARY INPUTS*
}

\author{
BY \\ J. L. BROWN, Jr. \\ (Ordnance Research Laboratory, The Pennsylvania State University)
}

1. Introduction. In [1], K. S. Miller obtained an expression for the mean-square output of a linear time-invariant filter subjected to a nonstationary random input, $y(t)=g(t) x(t)$, where $x(t)$ is a wide-sense stationary random process and $g(t)$ is a deterministic function of the particular form

$$
g(t)=\sum_{n=1}^{N} a_{n} \cos \left(\omega_{n} t+\phi_{n}\right) .
$$

The purpose of this note is to generalize Miller's result in two directions; we shall consider arbitrary modulation functions $g(t)$ and also obtain an expression for the general output autocorrelation function

$$
\phi_{z}\left(t_{1}, t_{2}\right)=E\left[z\left(t_{1}\right) z\left(t_{2}\right)\right],
$$

where $z(t)$ is the output corresponding to the input $y(t)=g(t) x(t)$. The mean-square output is then easily obtained by taking $t_{1}=t_{2}=t$. Since Miller's formula is immediate on further specialization of the modulation function, the general analysis affords as a by-product a much simpler proof of the result in [1]. In the concluding section, a reciprocity theorem is proved which shows that the mean-square output of a linear filter is invariant with respect to an interchange of the impulse response $h(t)$ and the modulation function $g(t)$.

In addition to the application suggested by Miller [1], we note that reverberation noise is commonly modeled [2] in the form $g(t) x(t)$, where $g(t)$ represents the timevarying decay characteristic of the reverberation and $x(t)$ is a stationary random process. Thus, nonstationary noises of the more general type considered here are involved in the analysis of sonar detection systems operating in a reverberation-limited environment.

2. Analysis. Let $y(t)=g(t) x(t)$ denote the input to a linear time-invariant system with impulse response $h(t)$, so that the output $z(t)$ is given by

$$
z(t)=\int_{-\infty}^{\infty} h(t-\xi) y(\xi) d \xi .
$$

We assume both $h(t)$ and $g(t)$ are real-valued with $g(t)$ uniformly bounded on $(-\infty, \infty)$ and $h(t)$ in the class $L_{1} \cap L_{2}$ on $(-\infty, \infty)$; that is, $h(t)$ is square integrable and corresponds to a stable filter so that it is also absolutely integrable. The real-valued random process $x(t)$ is assumed wide-sense stationary with square integrable autocorrelation function $\phi_{x}(\tau) \equiv E[x(t) x(t+\tau)]$ and related power spectral density $S_{x}(\omega)$.

Using (2) and noting that $E[y(\xi) y(\eta)]=g(\xi) g(\eta) \phi_{x}(\xi-\eta)$,

$$
\phi_{z}\left(t_{1}, t_{2}\right) \equiv E\left[z\left(t_{1}\right) z\left(t_{2}\right)\right]=\int_{-\infty}^{\infty} \int_{-\infty}^{\infty} h\left(t_{1}-\xi\right) h\left(t_{2}-\eta\right) g(\xi) g(\eta) \phi_{x}(\xi-\eta) d \xi d \eta .
$$

*Received August 27, 1965; revised manuscript received October 14, 1965. 
We next define a function $B(\omega, t)$ as follows:

$$
B(\omega, t)=\int_{-\infty}^{\infty} h(t-\xi) g(\xi) e^{-i \xi \omega} d \xi .
$$

Then, by Parseval's theorem [3], the $\xi$-integration in (3) becomes

$$
\int_{-\infty}^{\infty} h\left(t_{1}-\xi\right) g(\xi) \phi_{x}(\xi-\eta) d \xi=\frac{1}{2 \pi} \int_{-\infty}^{\infty} B\left(\omega, t_{1}\right) S_{x}(\omega) e^{i \eta \omega} d \omega
$$

and the remaining $\eta$-integration yields

$$
\phi_{z}\left(t_{1}, t_{2}\right)=\frac{1}{2 \pi} \int_{-\infty}^{\infty} B\left(\omega, t_{1}\right) B^{*}\left(\omega, t_{2}\right) S_{x}(\omega) d \omega,
$$

where an asterisk superscript denotes complex conjugate. Equation (5) with $B(\omega, t)$ given by (4) is the main result of this section. For $t_{1}=t_{2}=t$, equations (4) and (5) specialize to

$$
E\left[z^{2}(t)\right]=\frac{1}{2 \pi} \int_{-\infty}^{\infty}|B(\omega, t)|^{2} S_{x}(\omega) d \omega,
$$

which is the desired extension of Miller's result to arbitrary bounded modulation functions $g(t)$.

If, in addition, $g(t) \varepsilon L_{2}$ on $(-\infty, \infty)$, then by Parseval's theorem applied to (4), we have

$$
B(\omega, t)=\frac{1}{2 \pi} \int_{-\infty}^{\infty} H(\eta) G(\eta+\omega) e^{i \eta t} d \eta,
$$

where $H(\omega)$ and $G(\omega)$ are the $\left(L_{2}\right)$ Fourier transforms of $h(t)$ and $g(t)$ respectively. Equation (6) may then be written equivalently in terms of the spectra:

$$
E\left[z^{2}(t)\right]=\frac{1}{8 \pi^{3}} \int_{-\infty}^{\infty}\left|\int_{-\infty}^{\infty} H(\eta) G(\eta-\omega) e^{i \eta t} d \eta\right|^{2} S_{x}(\omega) d \omega .
$$

3. Miller's Theorem. To see that (6) contains the previous result [1] as a special case, we consider $g(t)$ to have the particular form

$$
g(t)=\sum_{n=1}^{N} a_{n} \cos \left(\omega_{n} t+\phi_{n}\right)
$$

then from (4),

$$
\begin{aligned}
B(-\omega, t) & =\frac{1}{2} \sum_{n=1}^{N} a_{n} \int_{-\infty}^{\infty} h(t-\xi)\left[e^{i\left(\omega_{n} \xi+\phi_{n}\right)}+e^{-i\left(\omega_{n} \xi+\phi_{n}\right)}\right] e^{i \omega \xi} d \xi \\
& =\frac{1}{2} e^{i \omega t}\left[M(\omega)+M^{*}(-\omega)\right],
\end{aligned}
$$

where we have followed Miller in defining

$$
M(\omega) \equiv \sum_{n=1}^{N} a_{n} H\left(\omega+\omega_{n}\right) e^{i\left(\omega_{n} l+\phi_{n}\right)} .
$$

Then, noting that $B(-\omega, t)=B^{*}(\omega, t)$, substitute (9) in (6) to obtain

$$
E\left[z^{2}(t)\right]=\frac{1}{8 \pi} \int_{-\infty}^{\infty}\left|M(\omega)+M^{*}(-\omega)\right|^{2} S_{x}(\omega) d \omega,
$$

which is easily seen to be the equivalent of Miller's formula (equation (4) in [1]). 
4. Reciprocity Theorem. In this section we require $g(t)$ [as well as $h(t)$ ] to belong to $L_{1} \cap L_{2}$ on $(-\infty, \infty)$. An obvious change of variable coupled with the observation that $S_{x}(\omega)$ is an even function allows us to write (8) in the alternate form:

$$
\begin{aligned}
E\left[z^{2}(t)\right] & =\frac{1}{8 \pi^{3}} \int_{-\infty}^{\infty}\left|\int_{-\infty}^{\infty} H(\eta) G(\eta+\omega) e^{i \eta t} d \eta\right|^{2} S_{x}(\omega) d \omega \\
& =\frac{1}{8 \pi^{3}} \int_{-\infty}^{\infty}\left|\int_{-\infty}^{\infty} H(\xi-\omega) G(\xi) e^{i(\xi-\omega) t} d \xi\right|^{2} S_{x}(\omega) d \omega .
\end{aligned}
$$

Rearranging the latter expression and changing the dummy variable back to $\eta$, we have

$$
E\left[z^{2}(t)\right]=\frac{1}{8 \pi^{3}} \int_{-\infty}^{\infty}\left|\int_{-\infty}^{\infty} G(\eta) H(\eta-\omega) e^{i \eta t} d \eta\right|^{2} S_{x}(\omega) d \omega .
$$

Comparison of (8) and (11) proves the following reciprocity result:

Let $x(t)$ be a wide-sense stationary random process with square integrable power spectral density $S_{x}(\omega)$. If $h(t)$ and $g(t)$ are two given real-valued functions in $L_{1} \cap L_{2}$ on $(-\infty, \infty)$, then the mean-square output of a time-invariant linear filter with impulse response $h(t)$ and input $y(t)=g(t) x(t)$ is exactly the same as the mean-square output of a linear system with impulse response $g(t)$ and input $y(t)=h(t) x(t)$.

Thus, the mean-square output of a linear system is invariant with respect to an interchange in roles of the impulse response $h(t)$ and the modulation function $g(t)$.

Lastly, we observe that the time dependent spectral density $W(t, \omega)$ defined by

$$
W(t, \omega) \equiv \int_{-\infty}^{\infty} \phi_{z}(t, t+\tau) e^{-i \omega \tau} d \tau
$$

can be calculated from (5) to give the following result:

$$
W(t, \omega)=\frac{e^{i \omega t} H(\omega)}{2 \pi} \int_{-\infty}^{\infty} B(\alpha, t) G(\omega-\alpha) S_{x}(\alpha) d \alpha .
$$

This formula generalizes equation (16) of Miller's paper [1] and can easily be shown to reduce to that result when $g(t)=\sum_{n=1}^{N} a_{n} \cos \left(\omega_{n} t+\phi_{n}\right)$. For the special case,

$$
G(\omega)=\pi \sum_{n=1}^{N} a_{n}\left[e^{i \phi_{n}} \delta\left(\omega-\omega_{n}\right)+e^{-i \phi_{n}} \delta\left(\omega+\omega_{n}\right)\right],
$$

where $\delta(\omega)$ is the delta functional and $B(\alpha, t)$ in (12) is given by $\left(e^{-i \alpha t} / 2\right)\left[M(-\alpha)+M^{*}(\alpha)\right]$ from equation (9).

\section{ReFerences}

1. K. S. Miller, A note on input-output spectral densities, Q. Appl. Math. 21, 249 (1963)

2. V. V. Ol'shevskii, Probability distribution of sea reverberation levels, Soviet Physics-Acoustics, 9, 378 (1964)

3. R. R. Goldberg, Fourier Transforms, Cambridge University Press, p. 48 (1961) 